\title{
Smart contracts - the new form of the legal agreements
}

\author{
Charlotte ENE \\ Bucharest University of Economic Studies, Bucharest, Romania \\ enecharlotte@gmail.com
}

\begin{abstract}
Today we are witnesses an explosion of online business, developed on the internet - a special environment that requires own resources and tools and it is governed by specific rules. In this context, a new type of technology has been developed - the distributed ledger system, which allowed the creation of a new form of the agreement - the smart contracts. Smart contract is the next step forward in the process of digitalized contracts, after using the PDF documents with electronic signatures, and it favors the businesses to be carried out completely automatically, without the need for human intervention, and to gain greater efficiency and reduction in costs. This paper will try to provide the answers to several questions, such as: what is a smart contract?; how smart contract will be used?; how smart contract will be enforced?; etc. Moreover, it will be emphasized the advantages of smart contract and the new developments such as "Ricardian" contracts representing more efficient and transparent agreements that can be drafted and enforced on platform. Most important issue of this paper consists in analysis of legal framework of smart contracts using the basic principles of contract law combined with blockchain regulations, taking into account changing the paradigm from "code is law" to "law is code".
\end{abstract}

Keywords: Smart Contracts, "Ricardian" Contracts, Wise contracts, Blockchain, Distributed Ledger System, Online Business, E-Commerce.

\section{Introduction}

The COVID-19 pandemic has revealed the importance of internet, as a workable medium for businesses, and other kind of activities. Thus, the online setting, with its own resources, tools and specific rules, requires an increasing attention in order to develop it, to improve the nexus between online and offline environments.

In this context, a new type of technology emerged - the distributed ledger system-, which allowed the creation of specific applications, including of a new form of the agreement - the smart contracts. Smart contract is the next step forward in the process of digitalized contracts, after using the PDF documents with electronic signatures (so called e-contracts), and it favors the businesses to be carried out completely automatically, without the need for human intervention, and to gain greater efficiency and reduction in costs.

This paper will try to provide the answers to several questions, such as: what is a smart contract?; how smart contract will be used?; how smart contract will be enforced?; etc. Moreover, it will be emphasized the advantages of smart contract and the new developments such as "Ricardian" contracts representing more efficient and transparent agreements that can be drafted and enforced on platform. Most important issue of this paper consists in analysis of legal framework of smart contracts using the basic principles of contract law combined with blockchain regulations, taking into account changing the paradigm from "code is law" to "law is code".

\section{The meaning of the "smart contract" concept}

The term "smart contract" is the creation of a computer scientist and cryptographer N. Szabo, who in the 1990s used it in connection with automatic vending machine.

According to that scientist, the automatic vending machine could be understood as a basic form of a smart contract, taking into account the fact that it distributes a specific good based on 
defined input data and preserves the ownership of the goods (sealed inside). Therefore, the vending machine can mechanically fulfill the obligations derived from the sale-purchase contract of the goods.

In his later papers, the above mentioned author generalized the concept of smart contract to any computer code which can record and perform transactions between parties, and defined it as: "Smart contracts combine protocols with user interfaces to formalize and secure relationships over computer networks. Objectives and principles for the design of these systems are derived from legal principles, economic theory, and theories of reliable and secure protocols." Moreover, smart contracts can "utilize protocols and user interfaces to facilitate all steps of the contracting process," such as: negotiation, concluding and performance. (N. Szabo, 1997)

Based on this approach, several direction of analysis of smart contract have been emerged from technical point of view, according to which smart contract is an autonomous code, an application of a distributed ledger technology (so called blockchain) (E. Mik, 2017) or a system "which automatically move digital assets according to arbitrary pre-specified rules." (B. Vitalik, 2015 ) to legal assertions which correlate digital concepts with legal terminology and self-execution of legal agreement, by using the computer code to perform contracts. (J. Stark, 2016).

Between these interpretations, smart contract is considered as being composed of computer protocols that "facilitate, verify, execute or embody the terms of a contract" (T. Swanson, 2014), or control assets by digital means (N. Szabo, 1997) thus having legal implications. (F. Zhang, et al., 2016).

Taking into account all these aspects, we agree with the definition of smart contract as an "electronic code that, upon the occurrence of (a) specified condition(s), is capable of running automatically according to pre-specified functions to execute a transaction between parties, stored and processed on a Blockchain or other distributed network and authenticated by a Digital Signature", provided by the Chamber of Digital Commerce based on both the legal and technical aspects of a smart contract. This definition for smart contracts is sufficiently broad to encompass many different types of transactions that parties may enter into.

So, smart contract is one of "the most promising benefits of the blockchain, (...) that capitalizes on blockchain features like immutability, accuracy, reliability, and transparency to ensure that transactions occur when pre-determined stipulations are met. (Confideal, 2017).

The main feature of the smart contract is the fact that it performs exactly what parties agree that they will do, meaning the self-execution of the agreement. This is the reason of the denomination of this kind of contracts as smart; parties may predetermine a set of conditions that are automatically performed when specific requirements are meet.

\section{Main traits of smart contract}

The application of smart contract is based on several essential traits, such as observability, verifiability, privity, and enforceability (N. Szabo, 1996), derived from the technical parameters of blockchain.

\section{Observability}

This element refers to the possibility of the contracting parties to observe each other's fulfillment of the contractual obligation, and/"'or to prove their performance to other principals." (N. Szabo, 1996)

The contracting parties aim that their agreement is executed according to the terms and conditions established from the conclusion. 
So, observability allows to either the other party or a third-party to objectively confirm the execution of the contract. In order to increase the observability, smart contracts are utilize separate computer programs (called "oracles") which find relevant data from outside the blockchain for the confirmation of party's performance. (BLOCKCHAINHUB, 2019).

\section{Verifiability}

The second basic trait of smart contracts represents the "ability of a principal to prove to an arbitrator that a contract has been performed or breached." (N. Szabo, 1996)

In the smart contract realm the third party is removed "because of the decentralized attributes of blockchain technology" (J. Arcari, 2018), therefore the verification become the duty of the contracting parties, a process "with minimal reliance on centrally provided systems or services.”(GITHUB, 2019)

\section{Privity}

The legal general principle of privity governs all type of contracts and it refers to the effects of the contract which are "produces only on its author or authors without being able to benefit or harm others." (O.I. Dumitru, A. Stoican, 2019)

However, the privity of the smart contracts implies the preservation of the privacy regarding the identity of the contracting parties or the terms and conditions of the transaction. (A. Kosba et al., 2016)

\section{(Self-)Enforceability}

The fourth trait consists in the ability of the smart contract to established mandatory obligations for the contracting parties and to put into execution based on sentence issued by the court of law.

Taking into account that smart contract is a digital code developed on a blockchain platform, it is automatically enforced on this distributed ledger system; thus, theoretically, it is no need for litigation surrounding missed expectations.

"Consequently, putting the smart contract "on" the blockchain ensures that the contractual obligations are executed without deviations because neither party can influence or interfere with its operation. Legal and commercial certainty are achieved by the fact that performance is technically guaranteed." (E. Mik, 2017)

The "self-enforcement", consisting in automated fulfillment of contractual obligation if the predetermined conditions are met, based on "if-then" action, represents the main advantage of smart contract and keeps it away from court room or any other third-party. (K.D. Atta-Krah, 2016)

On the other hand, the same trait - the self-execution- determines the limitations or disadvantages of smart contract. The execution of a smart contract is irreversible and parties cannot stop it or change it, "even if the performance of one of the parties is fraudulent of the terms of the agreement are later deemed to be void." (G. Fritz \& L. Treichl, 2019)

Moreover, being a computer code, the smart contract could be affected by software errors such as bugs and generate expansive problems for the contracting parties.

Briefly, "self-enforcement deprives contractual relationships of their adaptability and preclude the parties from adjusting their legal and commercial positions in response to changed circumstance;" (E. Mik, 2017) thus, a perfect execution of the contract it is not desirable (L.A. Shay et al., 2016) 


\section{Conclusion}

Obviously, the smart contract is in an incipient stage and have a lot of deficiencies. But, even at this moment we may see that this kind of contract is perfect for repetitive transactions, which are drawn up based on template slightly adapted. Moreover, when the contracting parties are fully agreed with each other, the smart contract is the perfect solution and the contractual terms proceed as planned. Before a general application of smart contract, it is important to be issued a uniform legal framework in this regard

\section{References}

Arcari, J., (2018). Smart Contracts-A Legal Contracts Perspective Part II: Verifiability, MEDIUM (Jul. 13, 2018), https://www.linkedin.com/pulse/smart-contractslegal-contractperspective-part-ii-jared-arcari/ [https://perma.cc/WZ69-QF2K].

Atta-Krah, K. D., (2016). Preventing A Boom from Turning Bust: Regulators Should Turn Their Attention to Starter Interrupt Devices Before the Subprime Auto Lending Bubble Bursts, 101 Iowa Law Review 1187

Dumitru, O.I., Stoican, A., (2019). Business Law - Lecture Notes, Ed. ASE, Bucuresti, 93.

Fritz, G. \& Treichl, L., (2019) What's in a Smart Contract? , FRESHFIELDS, BRUCKHAUS, DERINGER, https://www.freshfields.com/en-us/our-thinking/campaigns/digital/fintech/ whats-in/whats-in-a-smart-contract/ [https://perma.cc/SG8L-9V47]

Kosba, A., et al., (2016), Hawk: The Blockchain Model of Cryptography and Privacy-Preserving Smart Contracts, IEEE SYMP. SEC. \& PRIVACY 839, 839 https://ieeexplore.ieee.org/stamp/stamp.jsp?tp=\&arnumber=7546538 [https://perma.cc/6BAK-434N]

Mik, E. (2017). Smart Contracts: Terminology, Technical Limitations and Real World Complexity, downloaded from $\quad$ SSRN: https://ssrn.com/abstract=3038406, http://dx.doi.org/10.2139/ssrn.3038406.

Shay, L. A. et al., (2016). Confronting Automated Law Enforcement, in: R. Calo, M. Froomkin, I. Kerr eds., Robot Law, Edward Elgar, 258.

Stark, J. (2016). How Close Are Smart Contracts to Impacting Real-World Law? downloaded from www.coindesk.com/blockchain-smarts-contracts-real-world-law.

Szabo, N. (1996). Smart Contracts: Building Blocks for Digital Markets http://www.fon.hum.uva.nl/rob/Courses/InformationInSpeech/CDROM/Literature/LOT winterschool2006/szabo.best.vwh.net/smart_contracts_2.html [https://perma.cc/TQ5SNUWL].

Szabo, N. (1997). Smart Contracts: Formalizing and Securing Relationships on Public Networks 2 (9) First Monday 2.2.

Swanson, T. (2014). Great chain of numbers: A guide to smart contracts, smart property and trustless asset management, 11,16

Vitalik, B. (2015). Ethereum White Paper: A Next Generation Smart Contract \& Decentralized Application Platform.

Zhang, F. et al., (2016). Town Crier: An Authenticated Data Feed for Smart Contracts, 1.

BLOCKCHAINHUB, (2019). What's a Blockchain Oracle? Information Oracles \& External Data Feeds, https://blockchainhub.net/blockchain-oracles/ [https://perma.cc/NN Q3-M822].

Confideal, (2017). Smart Contracts Made Simple, https://www.newsbtc.com/2017/10/23/confideal-smart-contracts-made-simple. 
GITHUB, (2019). What is Smart Identity?, https://github.com/SmartIdentity/smartIdcontracts\#what-is-smart-identity [https://perma.cc/VM9C-6T8P].

Smart Contracts Alliance, (2018), Smart Contracts: Is the Law Ready? , CHAMBER DIGITAL COM., https://digitalchamber.org/smart-contracts-whitepaper/ [https://perma.cc/SAX8ZLH8].

PICBE | 1210 